\title{
Heat transport in nonlinear lattices free from the Umklapp process
}

\author{
Kazuyuki Yoshimura ${ }^{1}$, Yusuke Doi ${ }^{2}$, and Tomoya Kitamura ${ }^{3}$ \\ ${ }^{1}$ Faculty of Engineering, Tottori University, 4-101 Koyama-Minami, Tottori 680-8552, Japan \\ ${ }^{2}$ Division of Mechanical Engineering, Graduate School of Engineering \\ Osaka University, 2-1 Yamadaoka, Suita, Osaka 565-0871, Japan \\ ${ }^{3}$ Department of Information and Electronics, Graduate school of Engineering \\ Tottori University, 4-101 Koyama-Minami, Tottori 680-8552, Japan
}

\begin{abstract}
We construct one-dimensional nonlinear lattices having the special property such that the Umklapp process vanishes and only the normal processes are included in the potential functions. These lattices have long-range quartic nonlinear and nearest neighbor harmonic interactions with/without harmonic on-site potential. We study heat transport in two cases of the lattices with and without harmonic on-site potential by non-equilibrium molecular dynamics simulation. It is shown that the ballistic heat transport occurs in both cases, i.e., the scaling law $\kappa \propto N$ holds between the thermal conductivity $\kappa$ and the lattice size $N$. This result directly validates Peierls's hypothesis that only the Umklapp processes can cause the thermal resistance while the normal one do not.
\end{abstract}

PACS numbers: 05.45.-a, 44.10.+1

\section{INTRODUCTION}

Heat transport is ubiquitous in nature. In macroscopic scale materials, it is well described by the Fourier's law $J=-\kappa \nabla T$, where $J$ and $\nabla T$ are the heat flux and the temperature gradient, respectively, and $\kappa$ is a constant called the thermal conductivity, whose value is determined by the material. Consider a one-dimensional shaped material with length $L$ which is kept with different temperatures at the both ends. The Fourier's law implies that the heat flux $J$ is attenuated as $J \propto L^{-1}$ with increasing $L$, under a given temperature difference. This attenuation indicates the existence of thermal resistance. In microscopic scale, the value of $\kappa$ depends on both the material and its length [1], but the heat flux is still attenuated as the length increases, i.e., the thermal resistance still emerges. It has been a long-standing unsolved problem to clarify the origin of thermal resistance based on the dynamics of atoms.

A simple microscopic model for solids is one-dimensional lattice, and it has been used for studying heat transport via atomic vibrations [2, 3]. Nonlinearity of the lattice, which is necessary for the phonon interactions, is essential to explain the emergence of thermal resistance. There are two types of the phonon interaction processes, which are called the normal and the Umklapp processes. Peierls posed the hypothesis that only the Umklapp processes can cause the thermal resistance while the normal one do not [4, 5], and this hypothesis has been widely believed so far. However, at least in classical physics regime, the hypothesis does not have a firm theoretical basis. To the best of our knowledge, only the existing basis is that in a lattice with periodic boundary conditions the harmonic part of its total heat flux is conserved if there is no Umklapp process, provided that the lattice has no dispersion [5-7]. The assumption of no dispersion is never satisfied in one-dimensional lattices, and this is not a satisfactory basis for the hypothesis.

The above hypothesis has not yet been verified even by numerical simulations. The crucial reason is a lack of a nonlinear lattice model that is free from the Umklapp process. In the present paper, we construct a class of nonlinear lattices without the Umklapp process, which we call the Umklapp-free lattices (UFLs). The UFLs have long-range quartic nonlinear and nearest neighbor harmonic interactions with/without harmonic on-site potential. They closely relate with the Pairwise Interaction Symmetric Lattice (PISL) [8, 9], which is a special lattice model recently constructed and having a hidden symmetry in its potential function to enhance the mobility of a localized mode called the discrete breather [10-13].

The UFL enables one to directly verify Peierls's hypothesis. We numerically study heat transport in two types of UFLs, which are with and without harmonic on-site potential, and show that the ballistic heat transport occurs in both of the UFLs, i.e., $\kappa \propto N$ holds between the thermal conductivity $\kappa$ and the lattice size $N$. Our results justify Peierls's hypothesis at least in the present lattice models.

We mention known results about heat transport in the PISL, as it is a model closely related to the UFL. A near ballistic transport, $\kappa \propto N^{\alpha}$ with $\alpha \simeq 1$, has been reported in some works [14-17], whereas a different value $\alpha \simeq 0.71$ in [18]. It is still unclear whether the PISL exhibits the ballistic transport or non-ballistic but anomalous one. However, at least, the PISL seems to have $\alpha$ significantly larger than nonlinear lattices which are known to exhibit anomalous heat transport such as the FPUT- $\alpha$ or $\beta$ lattices $(0.3 \lesssim \alpha \lesssim 0.4)$.

We emphasize a significance of our model from the point of view of future studies. The UFL is expected to be a good starting point to study the mechanism of emerging of thermal resistance. It is possible to gradually introduce the Umklapp processes into the UFL by perturbing its potential functions. Therefore, the mechanism may be clarified by numerically observing what kind of elementary process is occurring in the perturbed UFL, i.e., by identifying the scatterer and scattering process of phonons.

This paper is organized as follows. In Sec. II we describe the UFL model. In Sec. III we describe details of our numerical simulation of heat transport in the UFL. In Sec. IV] we show numerical results of the simulation. Finally, conclusions are drawn in Sec. V. 


\section{UMKLAPP-FREE LATTICE MODEL}

The model we constructed is a class of infinite lattices with long-range nonlinear interactions which is described by the Hamiltonian

$$
\begin{aligned}
H= & \sum_{n=-\infty}^{\infty} \frac{1}{2} p_{n}^{2}+\sum_{n=-\infty}^{\infty}\left[\frac{\mu_{0}}{2} q_{n}^{2}+\frac{\mu_{1}}{2}\left(q_{n+1}-q_{n}\right)^{2}\right] \\
& +\beta \sum_{n=-\infty}^{\infty} \sum_{r=1}^{\infty} \frac{1}{4 r^{2}}\left\{q_{n+r}-(-1)^{r} q_{n}\right\}^{4} .
\end{aligned}
$$

This model corresponds to a one-dimensional chain of unitmass particles, where $n$th particle has its position $x_{n}=a n+q_{n}$ given by the lattice spacing constant $a$ and the relative displacement $q_{n}$. In Eq. (1), $p_{n}$ is the momentum of $n$th particle, $\mu_{0}$ and $\mu_{1}$ are coefficients of the harmonic on-site and interaction potentials, and $\beta>0$ is the nonlinearity strength. Arbitrary non-negative values are possible for $\mu_{0}$ and $\mu_{1}$. The coupling strength between the $r$ th neighboring particles is proportional to $1 / r^{2}$. We call this lattice the UFL. Note that the UFL should be regarded as only a mathematically idealized model since the nonlinear interaction term in Eq. (1) is physically unnatural due to the factor $(-1)^{r}$.

The equations of motion derived from the Hamiltonian (1) are given by

$$
\begin{aligned}
\ddot{q}_{n} & =-\mu_{0} q_{n}+\mu_{1}\left(q_{n+1}-2 q_{n}+q_{n-1}\right) \\
& +\beta \sum_{r=1}^{\infty} \frac{1}{r^{2}}\left[\left\{(-1)^{r} q_{n+r}-q_{n}\right\}^{3}-\left\{q_{n}-(-1)^{r} q_{n-r}\right\}^{3}\right],
\end{aligned}
$$

where $n \in \mathbb{Z}$. Note that the total momentum $\sum_{n=-\infty}^{\infty} p_{n}$ is not conserved by Eq. (2) regardless of the value of $\mu_{0}$, as shown in Appendix $\mathrm{A}$

Define the normal mode coordinates $U(k)$ via the discrete Fourier transformation

$$
U(k)=\frac{1}{\sqrt{2 \pi}} \sum_{n=-\infty}^{\infty} q_{n} e^{-i k n},
$$

where we restrict the range of wavenumber $k$ to the first Brillouin zone, i.e., $k \in \mathbb{T} \equiv(-\pi, \pi]$. If we rewrite Eq. (2) in terms of $U(k)$, we can obtain the equation

$$
\begin{aligned}
\ddot{U}(k)+v_{k}^{2} U(k) & =\frac{4 \beta}{\pi} \int_{\mathbb{T}^{3}} d k_{1} d k_{2} d k_{3} \phi_{0}\left(k_{1}, k_{2}, k_{3}, k\right) \\
& \times U\left(k_{1}\right) U\left(k_{2}\right) U\left(k_{3}\right) \delta\left(k_{1}+k_{2}+k_{3}-k\right),
\end{aligned}
$$

where $U(k)$ depends on time $t, \phi_{0}$ is a time-independent function of $\left(k_{1}, k_{2}, k_{3}, k\right), \delta$ is Dirac's delta function, and $v_{k}^{2}$ is given by

$$
v_{k}^{2}=\mu_{0}+4 \mu_{1} \sin ^{2}(k / 2) .
$$

Details of the derivation of Eq. (4) are described in Appendix B

Ordinary one-dimensional lattices with quartic potentials have the mode couplings specified by both $k_{1}+k_{2}+k_{3}-k=0$ and $\pm 2 \pi$ (cf. Appendix B 1). The former $k_{1}+k_{2}+k_{3}-k=0$ is

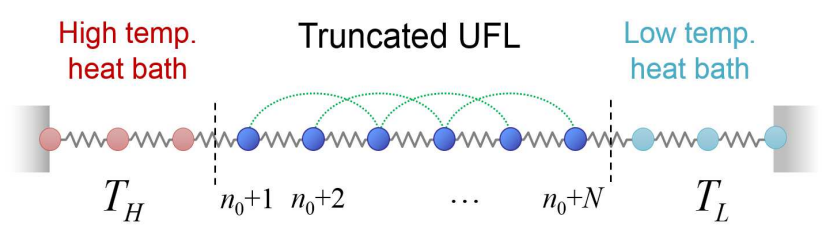

FIG. 1: Illustration of simulation model. Green dotted line represents the long-range interaction, where $M=2$ case is illustrated.

called the normal process while the latter $k_{1}+k_{2}+k_{3}-k= \pm 2 \pi$ the Umklapp process. Equation (4) shows that four normal modes are coupled only when their wavenumbers satisfy the condition $k_{1}+k_{2}+k_{3}-k=0$ while the couplings of $\pm 2 \pi$ are not allowed. This mode coupling rule is a peculiarity of the UFL, and it indicates the non-existence of the Umklapp process. As mentioned in Sec. [ the UFL closely relates with the PISL. Their relation is described in Appendix C.

\section{SIMULATION SETUP}

In order to study the heat transport by non-equilibrium molecular dynamics simulation, we introduce an approximate version of the UFL which has truncated long-range interactions up to length $M$, which we call the truncated UFL. This lattice is not exactly free from the Umklapp processes: the mode coupling terms specified by $k_{1}+k_{2}+k_{3}-k= \pm 2 \pi$ appear in its equation of motion in the normal mode coordinates $U(k)$. Those Umklapp terms become smaller and vanish as $M \rightarrow+\infty$. Thus, the truncated UFL satisfies the condition of non-existence of the Umklapp process in good approximation when $M$ is large enough.

In numerical simulations, we use a finite-size truncated UFL equipped with stochastic Langevin thermostats in its both ends. The equations of motion of our simulation model are given as follows:

$$
\ddot{q}_{n}=\lambda\left(q_{n+1}-2 q_{n}+q_{n-1}\right)-\gamma \dot{q}_{n}+\zeta_{n}(t)
$$

for $n \in I_{H} \cup I_{L}$ and

$$
\begin{aligned}
\ddot{q}_{n} & =-\mu_{0} q_{n}+\mu_{1}\left(q_{n+1}-2 q_{n}+q_{n-1}\right) \\
& +\beta \sum_{r=1}^{M} \frac{1}{r^{2}}\left[\left\{(-1)^{r} q_{n+r}-q_{n}\right\}^{3}-\left\{q_{n}-(-1)^{r} q_{n-r}\right\}^{3}\right]
\end{aligned}
$$

for $n \in I$, where $I_{H}=\left\{1,2, \ldots, n_{0}\right\}$ and $I_{L}=\left\{N+n_{0}+\right.$ $\left.1, \ldots, N+2 n_{0}\right\}$ are the sets of indices of particles equipped with high and low temperature thermostats, respectively, and $I=\left\{n_{0}+1, \ldots, n_{0}+N\right\}$ is the set of indices for the truncated UFL. The constant $\lambda$ can be different from $\mu_{1}$, but we assume $\lambda=\mu_{1}$ in the present simulation for simplicity. The range of nonlinear interactions is truncated up to $M$ in the model. In addition, the sum in Eq. (7) is taken only for the terms $\left\{(-1)^{r} q_{n+r}-q_{n}\right\}^{3}$ satisfying $n+r \leq n_{0}+N$ and the terms $\left\{q_{n}-(-1)^{r} q_{n-r}\right\}^{3}$ satisfying $n-r \geq n_{0}+1$. This implies that 
the nearest neighbor harmonic coupling is assumed between $n_{0}$ and $n_{0}+1$ particles and between $n_{0}+N$ and $n_{0}+N+1$ particles, which are connections between the truncated UFL and the heat baths. As for the boundary conditions, we assume $q_{0}=q_{N+2 n_{0}+1}=0$. Figure 1 illustrates the simulation model.

In Eq. [6, $-\gamma \dot{q}_{n}+\zeta_{n}(t)$ represents the Langevin thermostat, where $\gamma>0$ is a constant and $\zeta_{n}(t)$ is the white Gaussian noise having the properties

$$
\begin{aligned}
& \left\langle\zeta_{n}(t)\right\rangle=0, \\
& \left\langle\zeta_{n}(t) \zeta_{m}(s)\right\rangle=2 \gamma T \delta_{n, m} \delta(t-s),
\end{aligned}
$$

where $\langle\cdot\rangle$ denotes the averaging over realizations of $\zeta_{n}(t), \delta_{n, m}$ is Kronecker's delta, and $\delta$ is Dirac's delta function. The parameter $T$ represents the thermostat temperature, which is set as $T=T_{H}$ and $T_{L}$ for the high and low temperature sides, respectively.

The heat flux can be measured via a simple expression at the boundaries of the truncated UFL, i.e., $n=n_{0}+1$ and $n_{0}+N$ particles. Each of these two particles is coupled with its nearest neighbor thermostated particle via harmonic interaction force only. Noting this fact, we obtain the expression for the heat flux $J_{1}$, which is the energy transported from $n_{0}$ th particle to $\left(n_{0}+1\right)$ th one per unit time, as follows:

$$
J_{1}=-\left\langle\dot{q}_{n_{0}+1} \cdot \mu_{1}\left(q_{n_{0}+1}-q_{n_{0}}\right)\right\rangle_{\tau},
$$

where $\langle\cdot\rangle_{\tau}$ represents averaging over a long time $\tau$, i.e., $\langle X\rangle_{\tau}=$ $\tau^{-1} \int_{0}^{\tau} X(t) d t$ for an arbitrary quantity $X(t)$. Similarly, we can obtain the heat flux $J_{2}$ at $\left(n_{0}+N\right)$ th particle as follows:

$$
J_{2}=-\left\langle\dot{q}_{n_{0}+N} \cdot \mu_{1}\left(q_{n_{0}+N+1}-q_{n_{0}+N}\right)\right\rangle_{\tau} .
$$

If we measure the heat flux at an inner particle of the truncated UFL with $n \in\left\{n_{0}+2, \ldots, n_{0}+N-1\right\}$, we will have a more complex expression of heat flux due to the long-range interactions. So, we chose the two boundary particles. In the simulation, we compute the heat flux $J$ by the average of $J_{1}$ and $J_{2}$, i.e.,

$$
J=\frac{1}{2}\left(J_{1}+J_{2}\right) .
$$

The thermal conductivity $\kappa$ is defined by

$$
\kappa=\frac{J}{\left(T_{H}-T_{L}\right) / N} .
$$

We will focus on the $N$-dependence of $\kappa$. It is well known that one-dimensional lattices exhibit the power law $\kappa \propto N^{\alpha}$ with $0 \leq \alpha \leq 1$ [7]. The heat transport is called normal when $\alpha=0$ while it is called anomalous when $\alpha>0$. In particular, it is called the ballistic heat transport when $\alpha=1$, and this implies the state of vanishing thermal resistance.

We introduce spectral energy flux to study the heat transport process in detail. If we neglect the nonlinear forces and only consider the harmonic one in Eq. (2), we can define the harmonic part of the total heat flux as follows:

$$
J_{H, t o t}=-\frac{\mu_{1}}{2} \sum_{n=n_{0}+1}^{n_{0}+N-1}\left(\dot{q}_{n+1}+\dot{q}_{n}\right)\left(q_{n+1}-q_{n}\right) .
$$

Let $u_{k} \in \mathbb{C}, k=-N / 2+1, \ldots, N / 2$ be the mode amplitudes defined by the transformation

$$
q_{n_{0}+n}=\frac{1}{\sqrt{N}} \sum_{k=-N / 2+1}^{N / 2} u_{k} \exp \left[-i \frac{2 \pi k}{N} n\right], n=1,2, \ldots, N,
$$

where $u_{-k}=\bar{u}_{k}$ holds since $q_{n_{0}+n} \in \mathbb{R} . \bar{u}_{k}$ stands for the complex conjugate of $u_{k}$. In terms of $u_{k}$, we can decompose $J_{H, t o t}$ into the form $J_{H, t o t}=\sum_{k=1}^{N / 2-1} J_{H}(k)$ with

$$
J_{H}(k)=2 \omega_{k} v_{k} \operatorname{Im}\left[\dot{u}_{k} \bar{u}_{k}\right], \quad k=0,1, \ldots, N / 2,
$$

where $\omega_{k}$ and $v_{k}$ are defined by $\omega_{k}=2 \sqrt{\mu_{1}}|\sin (\pi k / N)|$ and $v_{k}=\sqrt{\mu_{1}} \operatorname{sgn}(k) \cos (\pi k / N)$. This quantity $J_{H}(k)$ is the harmonic part of the net energy flux carried by two modes with wavenumbers $\pm k$. The derivation of Eq. 16] is described in Appendix D

\section{SIMULATION RESULTS}

We numerically solved Eqs. (6) and (7) to compute the thermal conductivity $\kappa$ for different lattice sizes $N$, by using the Verlet scheme with time step $\Delta t=0.05$. Computation of the long-range nonlinear interaction forces in Eq. (7) is time-consuming for large values of $M$. To overcome this difficulty, we utilized GPU (NVIDIA GeForce RTX3080) for high-speed computation. The parameter values used in the simulation are $\lambda=1, \gamma=0.2, T_{H}=1.2, T_{L}=0.8$, and $n_{0}=10$.

Figures 22(a) and 2(b) show the logarithmic plots of $\kappa$ as a function of $N$ for $\mu_{0}=0$ and $\mu_{0}=1$ cases, i.e, the lattices without and with harmonic on-site potential, respectively. The weakly nonlinear case of $\mu_{1}=1$ and $\beta=0.1$ is assumed. The numerical results are shown for different values of the coupling length $M$ from $M=1$ to 512 .

In the simulations, we monitored the heat flux $J$ given by Eq. (12) as a function of $\tau$, which tends to converge to a constant as the averaging period $\tau$ increases. We used the convergence of $J(\tau)$ as a criterion for the system to have reached a steady state. In addition, we also monitored convergence of the spatial temperature profile (cf. Fig. 3).

In Fig. 2. a), the scaling of $\kappa$ with respect to $N$ precisely coincides with the ballistic one $\kappa \propto N$ over the whole range of simulation, i.e., up to $N=2^{20}$, in the case of $M=512$. For smaller values of $M$, the scaling is close to $\kappa \propto N$ as $N$ increases up to a certain value $N_{c}$, but it deviates from $\kappa \propto N$ as $N$ further increases. The values of $N_{c}$ are approximately found as $N_{c} \simeq 2^{19}, 2^{14}$, and $2^{11}$ for $M=256,64$, and 16 , respectively. $N_{c}$ decreases as $M$ decreases. In Fig.2(b), qualitatively the same behavior of $\kappa$ is observed. The ballistic transport is clearly observed for $M=512$ also in Fig. 2(b).

We are interested in the asymptotic scaling of $\kappa$ in the limit $N \rightarrow+\infty$, although numerical results are available only for finite $N$ values. An experimental formula is useful to infer the asymptotic scaling, and we have found it in the form

$$
\kappa=\frac{a N}{1+b N / M^{2}}
$$





FIG. 2: Thermal conductivity $\kappa$ vs. lattice size $N$ for truncated UFL $\left(\mu_{1}=1, \beta=0.1\right)$ (a) without on-site potential $\left(\mu_{0}=0\right)$ and (b) with on-site potential $\left(\mu_{0}=1\right)$. Results are shown by filled circles for different values of $M$. Temperatures are $T_{H}=1.2$ and $T_{L}=0.8$. Black dashed line represents the ballistic power law $\kappa \propto N$. Colored dashed lines are the fitting curves by experimental formula (17).

where $a$ and $b$ are the fitting parameters and their values are obtained as $a=0.1032, b=0.009904$ for $\mu_{0}=0$ and $a=$ $0.06314, b=0.004169$ for $\mu_{0}=1$, respectively. In Figs. 2 (a) and $2(\mathrm{~b})$, the curves of Eq. (17) with these values of $(a, b)$ are also shown. A good agreement between Eq. (17) and the numerical results is confirmed in each figure, except for the small $M$ cases of $M=1$ and 4 . This agreement suggests that it is a reasonable experimental formula at least for values of $M$ not too small. Once we accept Eq. 117, we can infer the behavior of $\kappa$ in the limit $N \rightarrow+\infty$. Equation (17) indicates that the asymptotic scaling $\kappa \propto N$ holds if we take the limit $N \rightarrow+\infty$ keeping the ratio $M / N$ as a constant.

As shown in Figs. 2(a) and 2(b), the truncated UFLs with $M=512$, where the non-existence of the Umklapp process holds in good approximation, exhibit the ballistic heat transport regardless of harmonic on-site potential. In contrast, in the cases of non-negligible Umklapp process, i.e., smaller $M$ cases, the ballistic heat transport breaks down for $N>N_{c}$. Based on these numerical observations and the inference via Eq. (17), it may be concluded that the thermal resistance is

\begin{tabular}{|c|c|c|}
\hline & Non-conserving & Conserving \\
\hline $\begin{array}{c}\text { Normal } \\
\alpha=0\end{array}$ & $\begin{array}{l}\text { Ding-a-ling [20,21] } \\
\text { Ding-dong [22] } \\
\text { Frenkel-Kontrova [23] } \\
\phi^{4} \text { chain }[24,25] \\
\text { Toda+harmonic on-site [26] }\end{array}$ & $\begin{array}{l}\text { Coupled rotators } \\
{[27]} \\
\text { Modified } \\
\text { ding-a-ling [28] }\end{array}$ \\
\hline $\begin{array}{c}\text { Anomalous } \\
0<\alpha<1\end{array}$ & & $\begin{array}{l}\text { FPUT- } \alpha[29] \\
\text { FPUT- } \beta[30,31] \\
\text { Diatomic Toda [32] }\end{array}$ \\
\hline $\begin{array}{c}\text { Ballistic } \\
\alpha=1\end{array}$ & UFL & Toda [33] \\
\hline
\end{tabular}

TABLE I: Classification of nonlinear lattice models by the type of heat transport and the total momentum conservation property.

never caused by the normal processes but only by the Umklapp one. That is, we have validated Peierls's hypothesis. We remark that it is not clear here whether all the Umklapp processes are resistive or only some of them are so.

One might expect the possibility that the ballistic transport in the UFL is caused simply by instantaneous energy transport over long distances via the long-range interaction forces. This issue has been studied for some nonlinear lattices with the long-range coupling coefficient $1 / r^{c}$ [19]. It has been shown that for $c>1$ such long-distance transport is non-dominant. This result is suggestive that the ballistic transport in UFL is being caused by the lack of Umklapp process.

The ballistic transport observed in the UFL is somewhat surprising from the fact that the total momentum is not conserved by Eq. (2). Table I summarizes known results for the type of heat transport and the total momentum conservation property in several one-dimensional nonlinear lattices. A common belief is that momentum non-conserving lattices belong to the class of normal heat transport, and this belief has been corroborated in various such lattices [20-26]. As Table I shows, all the momentum non-conserving lattices studied so far exhibit the normal heat transport, except for an example mentioned below. We emphasize that the UFL is a counter example against this common belief.

In Table I, we listed only nonlinear lattices of the natural Hamiltonian type, i.e., $H=\sum p_{n}^{2} / 2+V\left(q_{1}, \ldots, q_{N}\right)$. Other than this type, the ballistic transport has been reported for the Izergin-Korepin discrete sine-Gordon model, which is an integrable and momentum non-conserving model [34]. We also mention that there is a momentum-conserving coupled map lattice which exhibits the normal transport [35]. This model was derived from a Hamiltonian system with periodic impulsive kicks.

The PISL is momentum non-conserving when it has a harmonic on-site potential, otherwise it is momentum conserving. Its heat transport property has been studied for the momentum conserving PISL in [14, 15, 17, 18] while for both types of PISLs in [16]. Scaling laws close to the ballistic transport, i.e., $\kappa \propto N^{\alpha}$ with $\alpha \simeq 1$, are obtained in [14-17], whereas a different value $\alpha \simeq 0.71$ is reported in [18]. Reasons for this discrepancy in $\alpha$ are discussed in [17]. At this 

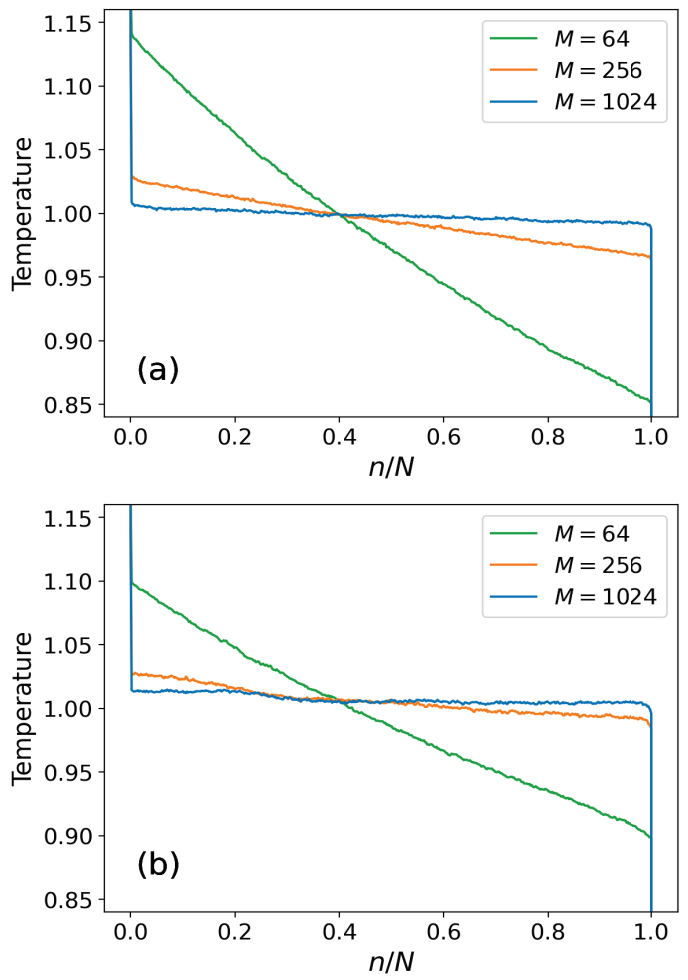

FIG. 3: Temperature profile plotted vs. $n / N$ for the truncated UFL $\left(\mu_{1}=1, \beta=0.1\right)$ (a) without on-site potential $\left(\mu_{0}=0\right)$ and (b) with on-site potential $\left(\mu_{0}=1\right)$. Profiles are shown for $M=64,256$, and 1024. Parameters are $N=2^{20}, T_{H}=1.2$, and $T_{L}=0.8$.

point, a definitive conclusion has not been settled in about the value of $\alpha$, and it is unclear whether the PISLs exhibit the ballistic transport or non-ballistic but anomalous one. So, we did not include the PISL in Table I.

Figures 3 a) and 3 b) show the spatial profile of temperature $T$ as a function of $n / N$ for $\mu_{0}=0$ and 1 , respectively, where $N=2^{20}, n$ is the site number, and the local temperature $T$ is defined by the time average of kinetic energy, i.e., $T=\left\langle p_{n}^{2}\right\rangle_{\tau}$. The results are shown for $M=64,256$, and 1024 . Apart from steep temperature variation in the regions close to the heat baths, the temperature gradient becomes smaller as $M$ increases, and the flat profile is formed for $M=1024$, in each figure. This flat profile is one of the characteristics of the ballistic heat transport.

The harmonic energy flux $J_{H}$ is plotted against $k / N$ for $\mu_{0}=0$ and 1 in Fig. 4 for different $N$, respectively, where $M=512$ is fixed. The other parameter values are the same as in Fig. 2 This figure indicates that contribution of the nonlinearity in heat transport is substantial since the profiles of $J_{H}$ are much different between the truncated UFLs and the harmonic lattices. This fact confirms that the ballistic transport observed in Fig. 2 is not due to a predominance of the linearity. The curves of $J_{H}(k / N)$ for different $N$ values coincide with each other in both cases of $\mu_{0}=0$ and 1 . The total amount of $J_{H}$ over the interval $k / N \in[0,0.5]$, which is defined by $\bar{J}_{H}=\int_{0}^{0.5} J_{H}(s) d s$ with $s=k / N$, is almost independent of

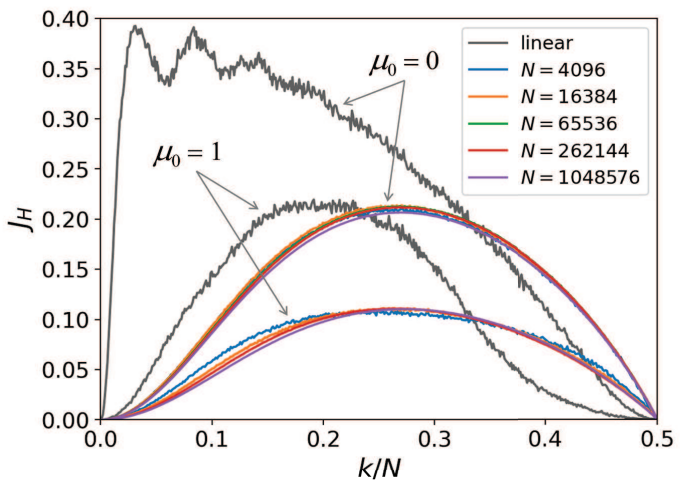

FIG. 4: Spectrum of harmonic energy flux $J_{H}$ plotted vs. $k / N$. Results are shown for truncated UFLs $\left(\mu_{1}=1, \beta=0.1\right)$ without onsite potential $\left(\mu_{0}=0\right)$ and with on-site potential $\left(\mu_{0}=1\right)$, where $M=512, T_{H}=1.2$, and $T_{L}=0.8$. Results are shown for $N=2^{12}$, $2^{14}, 2^{16}, 2^{18}$, and $2^{20} . J_{H}$ for harmonic lattices $\left(\mu_{1}=1, \beta=0\right)$ with $\mu_{0}=0$ and 1 are shown by black line for comparison.

$N$. This fact is consistent with the ballistic scaling $\kappa \propto N$. We note that $\bar{J}_{H}$ does not coincides with $J_{H, t o t}$ : they relate with each other as $\bar{J}_{H} \simeq J_{H, t o t} / N$. Comparing the profiles of curves of $J_{H}(k / N)$ between $\mu_{0}=0$ and 1 cases, there is a significant difference. This fact suggests that in our simulation the heat transport state is in actual influenced by the on-site potential, although only similar results are observed in Figs. 2] and 3 between $\mu_{0}=0$ and 1 cases.

Figure 4 shows that the normal modes over a broad range of $k / N$, especially over an intermediate range from $k / N \simeq 0.1$ to 0.45 , make non-negligible contributions to the heat transport. This shows that the heat transport mechanism of the UFL is quite different from that of the FPUT lattice, which exhibits the non-ballistic anomalous heat transport. In the FPUT lattice, only the normal modes with small $k$ make a dominant contribution while $J_{H}$ is strongly suppressed for the other larger $k$ as $N$ increases [31]. This suggests that those small $k$ modes form solitons and they induce the anomalous heat transport in the FPUT lattice. In contrast, $J_{H}$ is small for $k \simeq 0$, and this suggests that solitons are not formed and the normal modes, i.e., phonons, are the main heat carriers in the UFL. The heat transport by phonon is the situation supposed in Peierls's hypothesis.

In order to identify the thermal energy carriers precisely, we computed the space-time Fourier spectrum defined by

$$
S(k, \omega)=\frac{1}{\sqrt{\tau}} \int_{0}^{\tau} u_{k}(t) e^{-i \omega t} d t,
$$

where $u_{k}$ is the mode amplitude defined by Eq. 15 and $\tau$ is a time interval taken sufficiently long. Figure 5 shows the magnitude of $|S(k, \omega)|^{2}$ by color. There clearly appears a narrow strip-like curve indicated by bright red color, which represents large values, above the harmonic dispersion curve.

Equation (2) has the traveling wave solutions

$$
q_{n}(t)=A \cos (k n-\omega t), \quad n \in \mathbb{Z},
$$




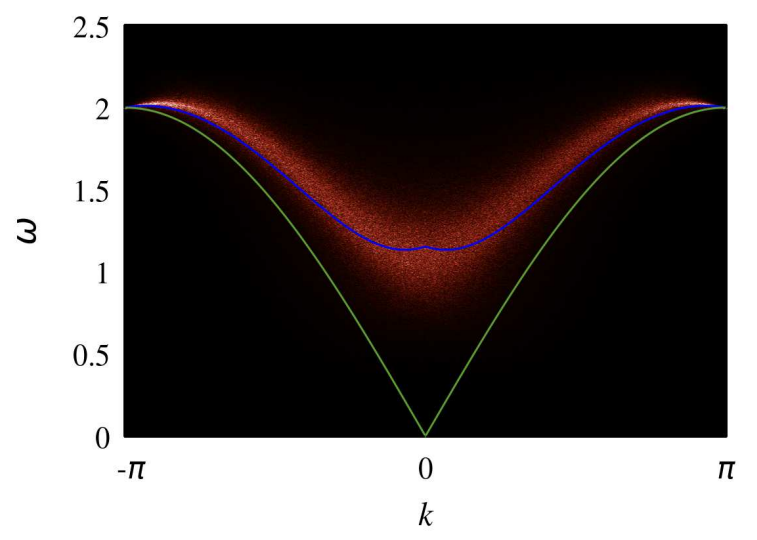

FIG. 5: Space-time Fourier spectrum of the steady heat transport state. $|S(k, \omega)|^{2}$ is presented by color. Parameters are $N=4096$, $\mu_{0}=0, \mu_{1}=1$, and $\beta=0.1$. Dispersion curve of the harmonic lattice $(\beta=0)$ and that of Eq. 20 are shown by green and blues lines, respectively.

where $\omega$ depends on $(k, A)$, and it is given by the nonlinear dispersion relation

$$
\omega=\sqrt{v_{k}^{2}+(3 / 2) \pi(\pi-|k|) \beta A^{2}},
$$

where $v_{k}^{2}$ is given by Eq. (5). We call this traveling wave the nonlinear phonon. The solution given by Eq. (19) is proved to be an exact one for $k \in[\pi / 3, \pi]$ in a similar manner to the proof in [15], while it is an approximate one for $k \in[0, \pi / 3)$.

The curve of Eq. 201 fitted to the numerical result by adjusting $A$ is shown by blue line in Fig. 5, and it is in good agreement with the narrow strip-like curve. A further numerical evidence is given in Appendix E Based on this agreement, we may conclude that the thermal energy is carried by the nonlinear phonons. Moreover, figure 5 indicates that the nonlinear phonons propagate with subsonic velocities, since their maximal group velocity $\max _{k \in[0, \pi]} \partial \omega / \partial k$, which can be estimated from the curve of Eq. (20) in Fig. 5, is smaller than the sound velocity $\partial v_{k} /\left.\partial k\right|_{k=0}$. We note that each of the nonlinear phonons does not propagate independently, but they exchange their energy via the normal processes during propagation, because superpositions of Eq. (19) are no longer exact or approximate solutions of Eq. (2).

\section{CONCLUSIONS}

We constructed nonlinear lattices having a special type of long-range quartic interaction potential such that the Umklapp process vanishes and only the normal processes exist, which we call the UFL. It is possible by using the UFL to directly verify Peierls's hypothesis that only the Umklapp processes can cause the thermal resistance while the normal one do not. Considering two types of the UFLs with and without the harmonic on-site potential, we studied their heat transport property by non-equilibrium molecular dynamics simulation. The numerical results and the experimental formula have shown that the ballistic heat transport, i.e., $\kappa \propto N$, occurs in the UFLs, and justify Peierls's hypothesis. Moreover, we pointed out the existence of the nonlinear phonons and showed that they are the thermal energy carriers which propagate with subsonic velocities. Finally, we emphasize that the UFL can be a good starting point to study the mechanism of emerging of the thermal resistance based on dynamics. It may be possible to clarify how the thermal resistance emerges via the Umklapp processes by perturbing the UFL.

\section{Acknowledgment}

The authors were supported by a Grant-in-Aid for Scientific Research (C), No. 19K03654 from Japan Society for the Promotion of Science (JSPS).

\section{Appendix A: Proof of non-conservation of total momentum}

We show non-conservation of the total momentum in Eq. (2). Precisely speaking, the total momentum $\sum_{n=-\infty}^{\infty} p_{n}$ of the infinite UFL does not necessarily have a finite value but may diverge. So, we employ its counterpart which is defined by a finite sum. Fix an arbitrary $N \in \mathbb{N}$, and impose the spatial periodicity condition $q_{n+N}=q_{n}, n \in \mathbb{Z}$ to Eq. (2). This is equivalent to considering a finite UFL consisting of $N$ particles under the periodic boundary condition, instead of the infinite UFL.

Let $M_{N}=\sum_{n=1}^{N} p_{n}$. We prove non-conservation of $M_{N}$. It can be checked that equation (2) has a solution of the form $q_{n}(t)=\phi(t), n \in \mathbb{Z}$, in which all the variables $q_{n}$ have the same displacement $\phi$. This solution apparently satisfies the spatial periodicity condition $q_{n+N}=q_{n}$. If we substitute this form into Eq. (2), we have the equation

$$
\ddot{\phi}=-\mu_{0} \phi-\sigma \phi^{3}
$$

where $\sigma=16 \beta \sum_{m=1}^{\infty}(2 m-1)^{-2}$. Equation A1 is regarded as that of a Hamiltonian oscillator with the potential $V(\phi)=$ $\mu_{0} \phi^{2} / 2+\sigma \phi^{4} / 4$, which is a single-well potential due to $\mu_{0} \geq$ 0 and $\sigma>0$. It is clear that this equation has a family of non-constant periodic solutions. Choose an arbitrary solution from the family. Along this solution, $\dot{\phi}(t)$ is a non-constant periodic function of $t$. This fact implies that $M_{N}=N \dot{\phi}(t)$ is not conserved. Thus, it has been proved that equation (2) does not conserve the total momentum in the sense that $M_{N}$ is not conserved for any $N \in \mathbb{N}$.

\section{Appendix B: Derivation of equation of motion in normal mode coordinates}

We describe derivation of Eq. (4) in the main text via two steps. In the first step, we consider a class of lattices with general quartic nonlinear interaction potentials, and derive its equation of motion in normal mode coordinates. In the second step, we assume the case of UFL and derive Eq. (4). 


\section{Normal mode equation for general nonlinear lattices}

Consider a class of infinite lattices described by the Hamiltonian

$$
\begin{aligned}
H_{\text {gen }}= & \sum_{n=-\infty}^{\infty} \frac{1}{2} p_{n}^{2}+\sum_{n=-\infty}^{\infty}\left[\frac{\mu_{0}}{2} q_{n}^{2}+\frac{\mu_{1}}{2}\left(q_{n+1}-q_{n}\right)^{2}\right] \\
& +\frac{\beta}{4} \sum_{n=-\infty}^{\infty} \sum_{r=1}^{\infty} b_{r}\left(q_{n+r}-\varepsilon^{r} q_{n}\right)^{4}
\end{aligned}
$$

where $\mu_{0}$ and $\mu_{1}$ are non-negative constants, $\beta>0$ is the nonlinearity strength, $b_{r}$ is the coupling strength between the $r$ th neighboring particles, and $\varepsilon \in\{-1,1\}$. This is a slightly generalized version of Hamiltonian (10), and it describes general nonlinear lattices with quartic two-body interactions. For instance, Hamitonian (B1) describes the UFL when $b_{r}=1 / r^{2}$ and $\varepsilon=-1$, while it describes the FPUT- $\beta$ lattice when $b_{r}=\delta_{r, 1}, \varepsilon=1$, and $\mu_{0}=0$, where $\delta_{r, 1}$ is Kronecker's delta.

The equations of motion derived from the Hamiltonian (B1) are given by

$$
\begin{aligned}
\ddot{q}_{n} & =-\mu_{0} q_{n}+\mu_{1}\left(q_{n+1}-2 q_{n}+q_{n-1}\right) \\
& +\beta \sum_{r=1}^{\infty} b_{r}\left[\left(\varepsilon^{r} q_{n+r}-q_{n}\right)^{3}-\left(q_{n}-\varepsilon^{r} q_{n-r}\right)^{3}\right],
\end{aligned}
$$

where $n \in \mathbb{Z}$.

The normal mode coordinates $U(k)$ are defined by the discrete Fourier transformation as follows:

$$
U(k)=\frac{1}{\sqrt{2 \pi}} \sum_{n=-\infty}^{\infty} q_{n} e^{-i k n}, \quad k \in(-\pi, \pi],
$$

where $k$ is restricted in the first Brillouin zone $\mathbb{T}=(-\pi, \pi]$. The inverse transformation is given by

$$
q_{n}=\frac{1}{\sqrt{2 \pi}} \int_{\mathbb{T}} U(k) e^{i k n} d k, \quad n \in \mathbb{Z} .
$$

Performing the discrete Fourier transformation to both sides of Eq. (B2), we have

$$
\begin{aligned}
& \ddot{U}(k)+v_{k}^{2} U(k)=\frac{\beta}{\sqrt{2 \pi}} \sum_{n=-\infty}^{\infty} e^{-i k n} \\
& \times \sum_{r=1}^{\infty} b_{r}\left[\left(\varepsilon^{r} q_{n+r}-q_{n}\right)^{3}-\left(q_{n}-\varepsilon^{r} q_{n-r}\right)^{3}\right],
\end{aligned}
$$

where $v_{k}^{2}=\mu_{0}+4 \mu_{1} \sin ^{2}(k / 2)$. Using Eq. (B4), we have

$$
\begin{aligned}
& \varepsilon^{r} q_{n+r}-q_{n}=\varepsilon^{r} \sqrt{\frac{2}{\pi}} \int_{\mathbb{T}} U(k) e^{i k n} e^{i r k / 2} g_{r}(k) d k, \\
& q_{n}-\varepsilon^{r} q_{n-r}=\sqrt{\frac{2}{\pi}} \int_{\mathbb{T}} U(k) e^{i k n} e^{-i r k / 2} g_{r}(k) d k,
\end{aligned}
$$

where $g_{r}(k)$ is given by

$$
g_{r}(k)=\frac{1}{2}\left(e^{i r k / 2}-\varepsilon^{r} e^{-i r k / 2}\right)= \begin{cases}\cos (r k / 2) & \text { for odd } r, \\ i \sin (r k / 2) & \text { for even } r .\end{cases}
$$

Substituting Eqs. B6 and (B7) into the right hand side of Eq. (B5), we have

$$
\begin{aligned}
\ddot{U}(k) & +v_{k}^{2} U(k) \\
= & \frac{\beta}{\sqrt{2 \pi}} \sum_{n=-\infty}^{\infty} e^{-i k n} \\
& \times \sum_{r=1}^{\infty} b_{r}\left[\prod_{j=1}^{3}\left\{\varepsilon^{r} \sqrt{\frac{2}{\pi}} \int_{\mathbb{T}} U\left(k_{j}\right) e^{i k_{j} n} e^{i r k_{j} / 2} g_{r}\left(k_{j}\right) d k_{j}\right\}\right. \\
& \left.-\prod_{j=1}^{3}\left\{\sqrt{\frac{2}{\pi}} \int_{\mathbb{T}} U\left(k_{j}\right) e^{i k_{j} n} e^{-i r k_{j} / 2} g_{r}\left(k_{j}\right) d k_{j}\right\}\right] \\
= & \frac{2 \beta}{\pi^{2}} \sum_{n=-\infty}^{\infty} \sum_{r=1}^{\infty} b_{r} \int_{\mathbb{T}^{3}} d k_{1} d k_{2} d k_{3} U\left(k_{1}\right) U\left(k_{2}\right) U\left(k_{3}\right) \\
& \times\left\{\varepsilon^{r} e^{i\left(k_{1}+k_{2}+k_{3}-k\right) r / 2} e^{i r k / 2}-e^{-i\left(k_{1}+k_{2}+k_{3}-k\right) r / 2} e^{-i r k / 2}\right\} \\
& \times G_{r}\left(k_{1}, k_{2}, k_{3}\right) e^{i\left(k_{1}+k_{2}+k_{3}-k\right) n},
\end{aligned}
$$

where $G_{r}$ is defined by

$$
G_{r}\left(k_{1}, k_{2}, k_{3}\right)=g_{r}\left(k_{1}\right) g_{r}\left(k_{2}\right) g_{r}\left(k_{3}\right) .
$$

As for the sum over $n$ in Eq. (B9), recall the formula

$$
\sum_{n=-\infty}^{\infty} e^{i n c x}=\frac{2 \pi}{c} \sum_{m=-\infty}^{\infty} \delta(x-2 \pi m / c)
$$

where $c, x \in \mathbb{R}$ are constants. We can calculate the sum over $n$ in Eq. (B9) by applying Eq. B11) with $c=1$ and $x=$ $k_{1}+k_{2}+k_{3}-k$. Then, we obtain

$$
\sum_{n=-\infty}^{\infty} e^{i\left(k_{1}+k_{2}+k_{3}-k\right) n}=2 \pi \sum_{m=-\infty}^{\infty} \delta\left(k_{1}+k_{2}+k_{3}-k-2 \pi m\right) .
$$

Using this and denoting $\lambda=k_{1}+k_{2}+k_{3}-k$, we can rewrite Eq. (B9) as follows:

$$
\begin{aligned}
\ddot{U}(k)+v_{k}^{2} U(k)= & \frac{4 \beta}{\pi} \sum_{r=1}^{\infty} b_{r} \int_{\mathbb{T}^{3}} d k_{1} d k_{2} d k_{3} U\left(k_{1}\right) U\left(k_{2}\right) U\left(k_{3}\right) \\
& \times\left\{\varepsilon^{r} e^{i(\lambda+k) r / 2}-e^{-i(\lambda+k) r / 2}\right\} G_{r}\left(k_{1}, k_{2}, k_{3}\right) \\
& \times \sum_{m=-\infty}^{\infty} \delta(\lambda-2 \pi m) .
\end{aligned}
$$

Since $-\pi<k_{j} \leq \pi$ and $-\pi \leq-k<\pi$, we have $-4 \pi<\lambda<$ $4 \pi$. Thus, there are only three possible values of $\lambda$, i.e., $\lambda=$ $0, \pm 2 \pi$, which correspond to $m=0, \pm 1$, respectively. Taking into account this fact, we can rewrite Eq. (B13) as

$$
\begin{aligned}
\ddot{U}(k)+v_{k}^{2} U(k)= & \frac{4 \beta}{\pi} \int_{\mathbb{T}^{3}} d k_{1} d k_{2} d k_{3} U\left(k_{1}\right) U\left(k_{2}\right) U\left(k_{3}\right) \\
& \times \sum_{m=-1}^{1} \delta(\lambda-2 \pi m) \phi_{m}\left(k_{1}, k_{2}, k_{3}, k\right),
\end{aligned}
$$

where $\phi_{m}$ is defined by

$$
\begin{aligned}
\phi_{m}\left(k_{1}, k_{2}, k_{3}, k\right)= & \sum_{r=1}^{\infty} b_{r}\left\{\varepsilon^{r} e^{i \pi m r} e^{i r k / 2}-e^{-i \pi m r} e^{-i r k / 2}\right\} \\
& \times G_{r}\left(k_{1}, k_{2}, k_{3}\right) .
\end{aligned}
$$




\section{Normal mode equation for UFL}

Hereafter, we assume the case of UFL, i.e., $b_{r}=1 / r^{2}$ and $\varepsilon=-1$, and derive Eq. (4). If we use $e^{i \pi m r}=e^{-i \pi m r}$, which follows from $m=0, \pm 1$, and denote $k^{\prime}=-k$, then we have

$$
\begin{aligned}
\phi_{m}\left(k_{1}, k_{2}, k_{3},-k^{\prime}\right)= & \sum_{r=1}^{\infty} \frac{1}{r^{2}}\left\{(-1)^{r} e^{-i k^{\prime} r / 2}-e^{i k^{\prime} r / 2}\right\} e^{i \pi m r} \\
& \times G_{r}\left(k_{1}, k_{2}, k_{3}\right) .
\end{aligned}
$$

Let $a=k_{1} / 2, b=k_{2} / 2, c=k_{3} / 2$, and $d=k^{\prime} / 2$. If we divide the sum in Eq. (B16) into two parts and use Eqs. (B8) and (B10), then we obtain

$$
\phi_{m}\left(k_{1}, k_{2}, k_{3},-k^{\prime}\right)=K_{o}(m)+K_{e}(m),
$$

where $K_{o}(m)$ and $K_{e}(m)$ are given by

$$
\begin{aligned}
& K_{o}(m)=-2 \sum_{r=\text { odd }} \frac{(-1)^{m}}{r^{2}} \cos (r a) \cos (r b) \cos (r c) \cos (r d), \\
& K_{e}(m)=-2 \sum_{r=\text { even }} \frac{1}{r^{2}} \sin (r a) \sin (r b) \sin (r c) \sin (r d) .
\end{aligned}
$$

The sums in Eqs. (B18) and (B19) are taken over all odd and even $r \in \mathbb{N}$, respectively.

We want to show $\phi_{m}\left(k_{1}, k_{2}, k_{3},-k^{\prime}\right)=0$ under the condition $k_{1}+k_{2}+k_{3}+k^{\prime}=2 \pi m$ for $m= \pm 1$. It is easy to see that $\phi_{m}$ has the property

$$
\phi_{m}\left(k_{1}, k_{2}, k_{3},-k^{\prime}\right)=\phi_{-m}\left(-k_{1},-k_{2},-k_{3}, k^{\prime}\right) .
$$

Because of this property, if $\phi_{m}\left(k_{1}, k_{2}, k_{3},-k^{\prime}\right)=0$ holds for any $\left(k_{1}, k_{2}, k_{3}, k^{\prime}\right)$ satisfying $k_{1}+k_{2}+k_{3}+k^{\prime}=2 \pi m$, then $\phi_{-m}\left(k_{1}, k_{2}, k_{3},-k^{\prime}\right)=0$ also holds for any $\left(k_{1}, k_{2}, k_{3}, k^{\prime}\right)$ satisfying $k_{1}+k_{2}+k_{3}+k^{\prime}=-2 \pi m$. Thus, it is enough to consider one of the $m= \pm 1$ cases. In what follows, we show $\phi_{1}\left(k_{1}, k_{2}, k_{3},-k^{\prime}\right)=0$.

A simple calculation using Eqs. (B18) and (B19) leads to

$$
\begin{aligned}
K_{o}(m)= & -\frac{1}{4} \sum_{r=\text { odd }} \frac{(-1)^{m}}{r^{2}}\{\cos (r(a+b+c+d)) \\
& +\cos (r(a-b+c+d))+\cos (r(a+b-c+d)) \\
& +\cos (r(a+b+c-d))+\cos (r(a+b-c-d)) \\
& +\cos (r(a-b+c-d))+\cos (r(a-b-c+d)) \\
& +\cos (r(a-b-c-d))\}, \\
K_{e}(m)= & -\frac{1}{4} \sum_{r=\text { even }} \frac{1}{r^{2}}\{\cos (r(a+b+c+d)) \\
& -\cos (r(a-b+c+d))-\cos (r(a+b-c+d)) \\
& -\cos (r(a+b+c-d))+\cos (r(a+b-c-d)) \\
& +\cos (r(a-b+c-d))+\cos (r(a-b-c+d)) \\
& -\cos (r(a-b-c-d))\} .
\end{aligned}
$$

Assuming $m=1$ and substituting Eqs. (B21) and B22) into Eq. (B17), we obtain

$$
\begin{aligned}
& \phi_{1}\left(k_{1}, k_{2}, k_{3},-k^{\prime}\right) \\
& =\frac{1}{4} \sum_{r=1}^{\infty} \frac{(-1)^{r-1}}{r^{2}}\{\cos (r(a+b+c+d))+\cos (r(a+b-c-d)) \\
& \quad+\cos (r(a-b+c-d))+\cos (r(a-b-c+d))\} \\
& +\frac{1}{4} \sum_{r=1}^{\infty} \frac{1}{r^{2}}\{\cos (r(a-b-c-d))+\cos (r(a-b+c+d)) \\
& \quad+\cos (r(a+b-c+d))+\cos (r(a+b+c-d))\} .
\end{aligned}
$$

Recall that $a, b, c \in(-\pi / 2, \pi / 2]$ and $d \in[-\pi / 2, \pi / 2)$, which follow from their definitions. We show $\phi_{1}=0$ for the wider range $a, b, c, d \in[-\pi / 2, \pi / 2]$. Since $\phi_{1}$ is invariant for any permutation of $a, b, c, d$, we can assume $\pi / 2 \geq a \geq b \geq c \geq$ $d \geq-\pi / 2$. Recall that $a+b+c+d=\pi$ holds when $m=1$. Note that (i) $a, b>0$ and (ii) $c \geq 0$ have to hold, because if $b \leq 0$ then $a \geq a+b+c+d=\pi$ and this contradict with $\pi / 2 \geq a$ and if $c<0$ then $a+b>a+b+c+d=\pi$ and this contradict with $\pi \geq a+b$. In addition, note that the sum of any pair taken from $\{a, b, c, d\}$ is positive, i.e., (iii) $x+y \geq 0$ for $x, y \in\{a, b, c, d\}$ such that $x \neq y$, because if $x+y<0$ then $z+w>x+y+z+w=a+b+c+d=\pi$ being in contradiction with $\pi \geq z+w$, where $w$ and $z$ are the elements other than $x$ and $y$.

Noting the properties (i)-(iii), we can evaluate the ranges of arguments of cosine functions in Eq. (B23) as follows:

$$
\begin{aligned}
& \text { In the first sum; } \\
& \begin{array}{l}
a+b+c+d=\pi, \\
0 \leq a+b-c-d=a+b-(c+d) \leq a+b \leq \pi \\
(\because a \geq c, b \geq d ; c+d \geq 0), \\
0 \leq a-b+c-d=a+c-(b+d) \leq a+c \leq \pi \\
\quad(\because a \geq b, c \geq d ; b+d \geq 0), \\
-\pi \leq-(b+c) \leq a-b-c+d \leq a+d \leq \pi \\
\quad(\because a+d \geq 0 ; b+c \geq 0),
\end{array}
\end{aligned}
$$

In the second sum;

$$
\begin{aligned}
0 & \leq-(a-b-c-d)=b+c+d-a=\pi-2 a \leq \pi \\
& (\because 0 \leq 2 a \leq \pi), \\
0 & \leq a-b+c+d=a+c+d-b=\pi-2 b \leq \pi \\
& (\because 0 \leq 2 b \leq \pi), \\
0 & \leq a+b-c+d=a+b+d-c=\pi-2 c \leq \pi \\
& (\because 0 \leq 2 c \leq \pi), \\
0 & \leq a+b+c-d=\pi-2 d \leq \pi+2|d| \leq 2 \pi \\
& (\because-\pi \leq 2 d \leq \pi) .
\end{aligned}
$$

In order to compute the two sums in Eq. (B23), recall the following formula

$$
\sum_{r=1}^{\infty} \frac{\cos r x}{r^{2}}=\frac{1}{4}(\varphi(x)-\pi)^{2}-\frac{\pi^{2}}{12},
$$

where $\varphi(x)$ is the function given by

$$
\varphi(x)=x-2 \pi l \text { for } x \in(2 \pi l, 2 \pi(l+1)], \quad l \in \mathbb{Z} .
$$


If we replace $x$ with $x+\pi$ in Eq. (B24), we can modify the above formula as follows:

$$
\sum_{r=1}^{\infty} \frac{(-1)^{r-1}}{r^{2}} \cos r x=\frac{\pi^{2}}{12}-\frac{x^{2}}{4}, \quad x \in[-\pi, \pi] .
$$

If we apply Eqs. (B24) and (B26) to Eq. B23 with noting the ranges of arguments of cosine functions, which were shown above, we have

$$
\begin{aligned}
& \phi_{1}\left(k_{1}, k_{2}, k_{3},-k^{\prime}\right) \\
= & \frac{1}{4} \cdot\left[\frac{\pi^{2}}{12}-\frac{\pi^{2}}{4}+\frac{\pi^{2}}{12}-\frac{(a+b-c-d)^{2}}{4}\right. \\
& +\frac{\pi^{2}}{12}-\frac{(a-b+c-d)^{2}}{4}+\frac{\pi^{2}}{12}-\frac{(a-b-c+d)^{2}}{4} \\
& +\frac{\{(b+c+d-a)-\pi\}^{2}}{4}-\frac{\pi^{2}}{12} \\
& +\frac{\{(a-b+c+d)-\pi\}^{2}}{4}-\frac{\pi^{2}}{12} \\
& +\frac{\{(a+b-c+d)-\pi\}^{2}}{4}-\frac{\pi^{2}}{12} \\
& \left.+\frac{\{(a+b+c-d)-\pi\}^{2}}{4}-\frac{\pi^{2}}{12}\right] \\
= & \frac{1}{16}(a+b+c+d)^{2}-\frac{\pi}{4}(a+b+c+d)+\frac{3}{16} \pi^{2} \\
= & 0,
\end{aligned}
$$

where we used $a+b+c+d=\pi$. Since it has been proved that $\phi_{ \pm 1}\left(k_{1}, k_{2}, k_{3},-k^{\prime}\right)=0$ when $k_{1}+k_{2}+k_{3}+k^{\prime}= \pm 2 \pi$, Equation B14 reduces to

$$
\begin{aligned}
\ddot{U}(k)+v_{k}^{2} U(k) & =\frac{4 \beta}{\pi} \int_{\mathbb{T}^{3}} d k_{1} d k_{2} d k_{3} \phi_{0}\left(k_{1}, k_{2}, k_{3}, k\right) \\
& \times U\left(k_{1}\right) U\left(k_{2}\right) U\left(k_{3}\right) \delta\left(k_{1}+k_{2}+k_{3}-k\right) .
\end{aligned}
$$

This equals to Eq. (4).

\section{Appendix C: Relation between UFL and PISL}

The PISL was originally constructed as a finite-size lattice with the periodic boundary condition [8, 9]. Its extension to the infinite-size one is described by the Hamiltonian

$$
\begin{aligned}
H= & \sum_{n=-\infty}^{\infty} \frac{1}{2} p_{n}^{2}+\sum_{n=-\infty}^{\infty}\left[\frac{\mu_{0}}{2} q_{n}^{2}+\frac{\mu_{1}}{2}\left(q_{n+1}-q_{n}\right)^{2}\right] \\
& +\beta \sum_{n=-\infty}^{\infty} \sum_{r=1}^{\infty} \frac{1}{4 r^{2}}\left(q_{n+r}-q_{n}\right)^{4}
\end{aligned}
$$

which corresponds to the case of $b_{r}=1 / r^{2}$ and $\varepsilon=1$ in Eq. (B1). The equations of motion are given by

$$
\begin{aligned}
\ddot{q}_{n}= & -\mu_{0} q_{n}+\mu_{1}\left(q_{n+1}-2 q_{n}+q_{n-1}\right) \\
& +\beta \sum_{r=1}^{\infty} \frac{1}{r^{2}}\left[\left(q_{n+r}-q_{n}\right)^{3}-\left(q_{n}-q_{n-r}\right)^{3}\right],
\end{aligned}
$$

where $n \in \mathbb{Z}$. If we fix an arbitrary even $N \in \mathbb{N}$ and impose the periodicity condition $q_{n+m N}=q_{n}, m \in \mathbb{Z}$ in Eq. (C2), it reduces to the equations of motion of the finite-size periodic PISL in [8, 9].

Equations (1) and (C1) show that nonlinear potentials of the UFL and the PISL are transformed to each other by the staggered transformation $q_{n} \rightarrow(-1)^{n} q_{n}$. In this sense, these two lattices correspond to each other. This correspondence implies that the two lattices have essentially the same dynamics when $\mu_{0}=\mu_{1}=0$, i.e., the homogeneous potential case.

Let us define $\tilde{U}(m)$ via the transformation

$$
\tilde{U}(m)=\frac{1}{\sqrt{2 \pi}} \sum_{n=-\infty}^{\infty}(-1)^{n} q_{n} e^{-i m n}, \quad m \in(-\pi, \pi],
$$

which is a composition of the staggered transformation and the discrete Fourier transformation defined by Eq. (B3). If we perform the above transformation to rewrite Eq. (C2) in terms of $\tilde{U}(m)$, its nonlinear force part can be computed in the same manner as in the UFL. Noting the linear force part, we obtain the equation

$$
\begin{aligned}
\ddot{\tilde{U}}(m)+\tilde{v}_{m}^{2} \tilde{U}(m) & =\frac{4 \beta}{\pi} \int_{\mathbb{T}^{3}} d m_{1} d m_{2} d m_{3} \phi_{0}\left(m_{1}, m_{2}, m_{3}, m\right) \\
& \times \tilde{U}\left(m_{1}\right) \tilde{U}\left(m_{2}\right) \tilde{U}\left(m_{3}\right) \delta\left(m_{1}+m_{2}+m_{3}-m\right),
\end{aligned}
$$

where $\phi_{0}$ is a time-independent function of $\left(m_{1}, m_{2}, m_{3}, m\right)$ and $\tilde{v}_{m}^{2}$ is given by

$$
\tilde{v}_{m}^{2}=\mu_{0}+4 \mu_{1} \cos ^{2}(m / 2)
$$

Equation (C4) has the same form as Eq. (4), but note that the dependence of $\tilde{v}_{m}^{2}$ on $m$ is different form that of $v_{k}^{2}$ on $k$. In the non-homogeneous potential case, the UFL and the PISL have different dynamics.

Equation (C4) shows that four normal modes are coupled only when their wavenumbers satisfy the condition $m_{1}+m_{2}+$ $m_{3}-m=0$ while the couplings of $\pm 2 \pi$ are not allowed. This mode coupling rule is a peculiarity of the PISL, which is similar to that of the UFL.

\section{Appendix D: Derivation of the spectral energy flux formula}

Consider the total harmonic heat flux $J_{H, t o t}$ given by Eq. (14). We approximate $J_{H, t o t}$ as follows:

$$
J_{H, t o t} \simeq-\frac{\mu_{1}}{2} \sum_{n=n_{0}+1}^{n_{0}+N}\left(\dot{q}_{n+1}+\dot{q}_{n}\right)\left(q_{n+1}-q_{n}\right) .
$$

where $q_{n_{0}+N+1}=q_{n_{0}+1}$. The sum is taken only up to $n=$ $n_{0}+N-1$ in the definition of $J_{H, t o t}$. In this approximation, we added the last term $-\mu_{1}\left(\dot{q}_{n_{0}+1}+\dot{q}_{n_{0}+N}\right)\left(q_{n_{0}+1}-q_{n_{0}+N}\right) / 2$. Since we assume large values of $N$ in our simulation, this last term is much smaller than the sum of other terms in Eq. (D1), and we can expect Eq. (D1) to be a good approximation. 
If we substitute Eq. (15) into Eq. (D1), we obtain

$$
\begin{aligned}
J_{H, t o t} & -\frac{\mu_{1}}{2 N} \sum_{n=1}^{N}\left\{\sum_{m=-N / 2+1}^{N / 2} \dot{u}_{m}\left(e^{-i \theta m}+1\right) e^{-i \theta m n}\right\} \\
& \times\left\{\sum_{k=-N / 2+1}^{N / 2} u_{k}\left(e^{-i \theta k}-1\right) e^{-i \theta k n}\right\} \\
= & -\frac{\mu_{1}}{2 N} \sum_{k, m=-N / 2+1}^{N / 2}\left[\sum_{n=1}^{N} \dot{u}_{m} u_{k}\left(e^{-i \theta m}+1\right)\left(e^{-i \theta k}-1\right) e^{-i \theta(k+m) n}\right],
\end{aligned}
$$

where $\theta=2 \pi / N$. Calculating the sum with respect to $n$, we have

$$
\begin{aligned}
J_{H, t o t}= & -\frac{\mu_{1}}{2 N} \sum_{k, m=-N / 2+1}^{N / 2}\left[\dot{u}_{m} u_{k}\left(e^{-i \theta m}+1\right)\left(e^{-i \theta k}-1\right)\right. \\
& \left.\times N\left(\delta_{k+m, 0}+\delta_{k+m, N}\right)\right]
\end{aligned}
$$

where $\delta_{k+m, 0}$ and $\delta_{k+m, N}$ are Kronecker's delta. The condition $k+m=N$ holds only for $k=m=N / 2$, and we have $e^{-i \theta m}+1=$ 0 in this case. If we calculate the sum with respect to $m$ in Eq. (D2), taking account of this fact, then we have

$$
\begin{aligned}
J_{H, t o t} & =-\frac{\mu_{1}}{2} \sum_{k=-N / 2+1}^{N / 2-1} u_{k} \dot{u}_{-k}\left(e^{i \theta k}+1\right)\left(e^{-i \theta k}-1\right) \\
& =i \mu_{1} \sum_{k=-N / 2+1}^{N / 2-1} u_{k} \dot{u}_{-k} \cdot 2 \sin \frac{\pi k}{N} \cos \frac{\pi k}{N} \\
& =i \sum_{k=-N / 2+1}^{N / 2-1} \omega_{k} v_{k} u_{k} \dot{u}_{-k},
\end{aligned}
$$

where $\omega_{k}=2 \sqrt{\mu_{1}}|\sin (\pi k / N)|$ and $v_{k}=\sqrt{\mu_{1}} \operatorname{sgn}(k) \cos (\pi k / N)$. Note that the term for $k=0$ vanishes due to $\omega_{0}=0$. Dividing the sum in Eq. (D3) into two parts, we can rewrite $J_{H, t o t}$ as follows:

$$
\begin{aligned}
J_{H, t o t} & =i \sum_{k=1}^{N / 2-1} \omega_{k} v_{k} u_{k} \dot{u}_{-k}+i \sum_{k=1}^{N / 2-1} \omega_{-k} v_{-k} u_{-k} \dot{u}_{k} \\
& =\frac{1}{i} \sum_{k=1}^{N / 2-1} \omega_{k} v_{k}\left(\dot{u}_{k} u_{-k}-u_{k} \dot{u}_{-k}\right) \\
& =\sum_{k=1}^{N / 2-1} 2 \omega_{k} v_{k} \operatorname{Im}\left[\dot{u}_{k} \bar{u}_{k}\right] \\
& =\sum_{k=1}^{N / 2-1} J_{H}(k),
\end{aligned}
$$

where we used $\omega_{-k} v_{-k}=-\omega_{k} v_{k}$ and $u_{-k}=\bar{u}_{k}$.

\section{Appendix E: Numerical evidence for nonlinear phonons}

In Fig. 5, the curve of $|S(k, \omega)|^{2}$ is not a sharp line but exhibits non-small line width. In addition, there is slight discrep- ancy between the dispersion curve of Eq. (20) and the average

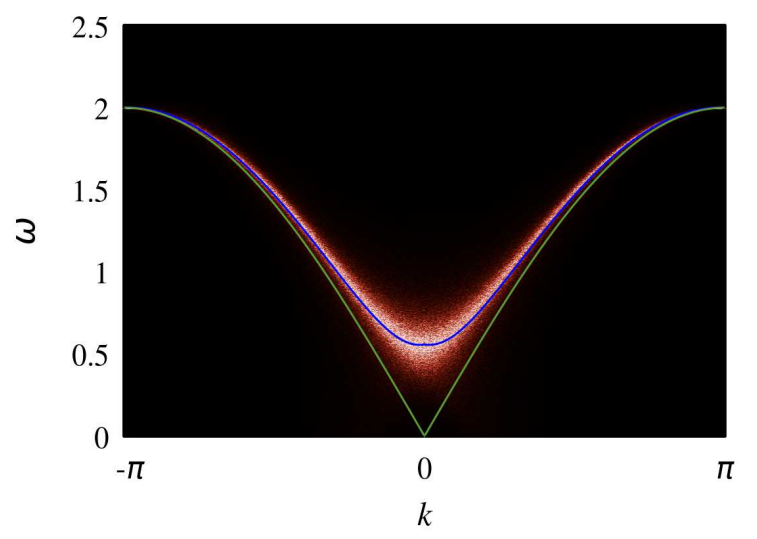

FIG. 6: Space-time Fourier spectrum of the steady heat transporting state in the UFL of weak nonlinearity $\beta=0.01$. $|S(k, \omega)|^{2}$ is presented by color. Parameters are $N=4096, \mu_{0}=0$, and $\mu_{1}=1$. Dispersion curve of the harmonic lattice $(\beta=0)$ and that of Eq. (20) are shown by green and blues lines, respectively.

profile of $|S(k, \omega)|^{2}$ curve, i.e., the middle line of its strip-like curve. Due to these facts, it might not be a fully convincing scenario that the nonlinear phonons emerge and carry the thermal energy in steady heat transport state. In this section, we give an additional numerical evidence to ensure this scenario.

We have assumed that the amplitude $A$ of nonlinear phonons is a constant independent of $k$ when fitting Eq. (20) to the numerical result of $|S(k, \omega)|^{2}$. However, in actual, it may be expected that the value of $A$ fluctuates in time and moreover the temporal average of $A$ depends on $k$, provided that the nonlinear phonons emerge. If we take into account these points, we may write the amplitude $A$ in the form

$$
A=A_{0}+\delta A(k)+\varepsilon(k, t)
$$

where $A_{0}$ is a constant, $A_{0}+\delta A(k)$ represents the temporal average of $A$ for $k$, and $\varepsilon(k, t)$ represents the temporal fluctuation in $A$ for a given $k$. This form may explain differences between the numerical result of $|S(k, \omega)|^{2}$ and the dispersion curve of Eq. 20): $\varepsilon(k, t)$ causes the line width and $\delta A(k)$ causes a deviation of the average profile from Eq. (20) under substitution of Eq. (E1) into Eq. (20).

The $A$-dependent term is in proportion to $\beta$ in Eq. (20). This fact suggests that the influences of $\delta A(k)$ and $\varepsilon(k, t)$ are small for small values of $\beta$, resulting in a better agreement of $|S(k, \omega)|^{2}$ with Eq. (20) for a $k$-independent constant $A$. Figure 6 shows $|S(k, \omega)|^{2}$ computed for the UFL of weak nonlinearity $\beta=0.01$, where the other parameters are the same as in Fig. 5. An excellent agreement is clearly observed. This agreement validates the above mentioned scenario. 
[1] C. W. Chang, D. Okawa, H. Garcia, A. Majumdar, and A. Zettl, Phys. Rev. Lett. 101, 075903 (2008).

[2] C. Kittel, Introduction to Solid State Physics (Wiley, 2004).

[3] N. Ashcroft and N. Mermin, Solid State Physics (Saunders College Publishing, New York, 1976).

[4] R. E. Peierls, Quantum Theory of Solids (Oxford University Press, London, 1955).

[5] R. E. Peierls, On the Kinetic Theory of Thermal Conduction in Crystals, in Selected Scientific Papers of Sir Rudolf Peierls, edited by R. H. Dalitz and R. Peierls (World Scientific, Singapore, 1997).

[6] E. A. Jackson, Rocky Mount. J. Math. 8, 127 (1978).

[7] S. Lepri, R. Livi, and A. Politi, Phys. Rep. 3771 (2003).

[8] Y. Doi and K. Yoshimura, Phys. Rev. Lett. 117, 014101 (2016).

[9] Y. Doi and K. Yoshimura, Nonlinearity 33, 5142 (2020).

[10] A. J. Sievers and S. Takeno, Phys. Rev. Lett. 61, 970 (1988).

[11] J. B. Page, Phys. Rev. B 417835 (1990).

[12] K. Yoshimura and Y. Doi, J. Diff. Eq. 298, 560 (2021).

[13] K. Yoshimura, Y. Doi, and M. Kimura, Localized modes in nonlinear discrete systems, in Progress in Nanophotonics III, edited by M. Ohtsu and T. Yatsui (Springer, New York, 2014), p. 119.

[14] D. Bagchi, Phys. Rev. E 95, 032102 (2017).

[15] K. Yoshimura and Y. Doi, Proc. of the 2019 Int. Symp. on Nonlinear Theory and Its Applications, 399 (2019).

[16] K. Yoshimura, Y. Doi, and M. Ebisu, Proc. of the 2020 Int. Symp. on Nonlinear Theory and Its Applications, 181 (2020).

[17] D. Bagchi, Phys. Rev. E 104, 054108 (2021).

[18] J. Wang, S. V. Dmitriev, and D. Xiong, Phys. Rev. Research 2 ,
013179 (2020).

[19] S. Iubini, P. Di Cintio, S. Lepri, R. Livi, and L. Casetti, Phys. Rev. E 97, 032102 (2018).

[20] G. Casati, J. Ford, F. Vivaldi, and W. M. Visscher, Phys. Rev. Lett. 52, 1861 (1984).

[21] D. J. R. Mimnagh and L. E. Ballentine, Phys. Rev. E 56, 5332 (1997).

[22] T. Prosen and M. Robnik, J. Phys. A 25, 3449 (1992).

[23] B. Hu, B. Li and H. Zhao, Phys. Rev. E 57, 2992 (1998).

[24] B. Hu, B. Li, H. Zhao, Phys. Rev. E 61, 3828 (2000).

[25] K. Aoki and D. Kusnezov, Phys. Lett. A 265, 250 (2000).

[26] P. D. Cintio, S. Iubini, S. Lepri, and R. Livi, Chaos, Solitons and Fractals 117, 249 (2018).

[27] C. Giardiná, R. Livi, A. Politi, and M. Vassalli, Phys. Rev. Lett. 84, 2144 (2000).

[28] G. R. Lee-Dadswell, E. Turner, J. Ettinger, and M. Moy, Phys. Rev. E 82, 061118 (2010).

[29] S. Lepri, European Physical Journal B 18, 441 (2000).

[30] S. Lepri, R. Livi, A. Politi, Physica D 119, 140 (1998).

[31] G. Dematteis, L. Rondoni, D. Proment, F. De Vita, and M. Onorato, Phys. Rev. Lett. 125, 024101 (2020).

[32] T. Hatano, Phys. Rev. E 59, R1 (1999).

[33] M. Toda, Physica Scripta 20, 424 (1979).

[34] T. Prosen and D. K. Campbell, Chaos 15, 015117 (2005).

[35] C. Giardinà and J. Kurchan, J. Stat. Mech. 2005, P05009 (2005). 